\title{
Model Predictive Control Of A Parafoil And Payload System
}

\author{
Nathan Slegers ${ }^{*} \quad$ Mark Costello $^{\dagger}$ \\ Department of Mechanical Engineering \\ Oregon State University \\ Corvallis, Oregon 97331
}

A model predictive control strategy is developed for an autonomous parafoil and payload system. Since the technique requires a linear dynamic model of the system, a reduced state linear model based on a nonlinear 6 degree-of-freedom parafoil and payload model is established and validated. In order to use the reduced state linear model for model predictive control the desired trajectory in the $x-y$ plane is mapped into a desired heading angle using Lagrange interpolating polynomials. Flight test results demonstrate that this model predictive control strategy is a natural and effective method of achieving trajectory tracking in a parafoil and payload system.

\section{Nomenclature}

$x, y, z=$ Components of position vector of the system mass center in an inertial frame.

$\phi, \theta, \psi=$ Euler roll, pitch and yaw angles of system.

$\dot{x}, \dot{y}, \dot{z}=$ Components of velocity vector of the system mass center in an inertial frame.

$p, q, r=$ Components of angular velocity of the system in the body reference frame.

$m_{T} \quad=$ Combined mass of payload and parafoil.

$I_{T} \quad=$ Inertia matrix of combined parafoil and payload system with respect to its mass center.

\footnotetext{
${ }^{*}$ Graduate Research Assistant, Department of Mechanical Engineering, Member AIAA.

${ }^{\dagger}$ Associate Professor, Department of Mechanical Engineering, Member AIAA.
} 


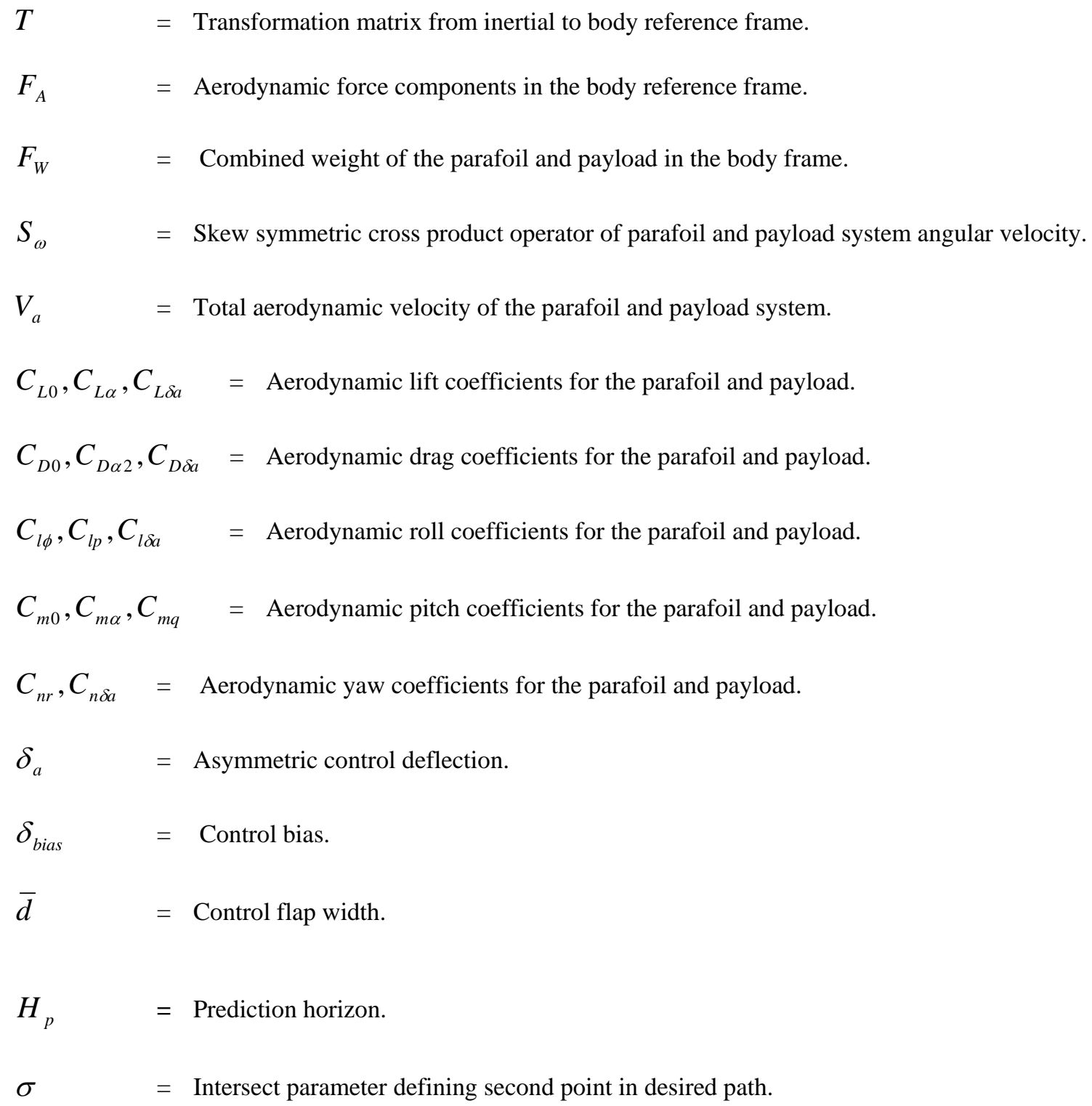

\section{Introduction}

$1 \mathrm{~N}$ air vehicle that is lightweight, flies at low speed, provides "soft" landing capability, and is compact before deployment is the parafoil and payload aircraft configuration. As the name suggests, the vehicle is comprised of a parafoil canopy connected to a payload body with suspension lines. Control is affected by two primary means, namely, deflection of left and right parafoil brakes and movement of the mass center of the complete system. Aviation enthusiasts commonly use these aircraft as recreational air vehicles. In this case, the pilot deflects the right and left parafoil brakes pulling down on the right and left side control lines. The pilot through appropriate 
body movement changes the center of mass of the system. The dynamics are sufficiently slow such that expert paraglider pilots can track a desired trajectory and attain accurate ground impact. Subconsciously these pilots continuously project the trajectory forward in time and compare the results with the desired path. The error between the projected and desired path is used to determine control action.

Parafoil and payload aircraft are also particularly well suited as an autonomous air vehicle for sensing applications. These air vehicles can be released at altitude from a parent delivery aircraft or can be hand launched from the ground. For autonomous control, each individual sensor payload is fitted with an inexpensive guidance and control module. A control strategy that mimics how human pilots control paragliders is model predictive control. In model predictive control, a dynamic model of the system is used to project the state into the future and subsequently use the estimated future states to determine control action. It is a common control technique in the process control industry. ${ }^{1}$ Currently, model predictive control is being applied to a wide variety of problems, spanning many different industries. Mei, Kareem, and Kontor studied vibration reduction of a tall building experiencing wind excitation using model predictive control and linear quadratic Gaussian control strategies. ${ }^{2}$ They found that the model predictive control scheme performed well and was robust to uncertainty in building stiffness. Tsai and Huang used a model reference adaptive predictive controller for a variable-frequency oil-cooling machine used with dynamically complex machine tool. ${ }^{3}$ Kvaternik, Piatak, Nixon, Langston, Singleton, Bennett, and Brown developed a generalized predictive controller for tilt rotor aeroelastic stability augmentation in airplane mode of flight. Using the model predictive control strategy, significant increases in damping of aircraft flexible vibration modes were achieved in a wind tunnel test. ${ }^{4}$ The work reported here creates a model predictive control strategy for a parafoil and payload aircraft. Performance of the autonomous flight control system is shown through flight tests of the system under a variety of conditions.

\section{Model Predictive Control}

Consider a discrete system described in state space form as given in Equation 1.

$$
\begin{aligned}
& x_{k+1}=A x_{k}+B u_{k}+D \\
& y_{k}=C x_{k}
\end{aligned}
$$


Assume that the system matrices $A, B, C$ and $D$ are known and that $x_{k}$ is the state vector, $u_{k}$ is the control input, and $y_{k}$ is the output at time $k$. The discrete model can be used to estimate the future state of the system. Assuming a desired trajectory is known $\left(w_{k}\right)$, an estimated error signal $\tilde{e}_{k}=w_{k}-\tilde{y}_{k}$ is computed over a finite set of future time instants called the prediction horizon, $H_{p}$. The symbol $\sim$ is used to represent an estimated quantity. In model predictive control, the control computation problem is cast as a finite time discrete optimal control problem. To compute the control input at a given time instant, a quadratic cost function is minimized through the selection of the control history over the control horizon. The cost function can be written as:

$$
J=(W-\tilde{Y})^{T}(W-\tilde{Y})+U^{T} R U
$$

where,

$$
\begin{gathered}
W=\left[\begin{array}{llll}
w_{k+1} & w_{k+2} & \cdots & w_{k+H_{p}}
\end{array}\right]^{T} \\
\tilde{Y}=K_{C A} X_{k}+K_{C A B} U+K_{C A D 1} \\
U=\left[\begin{array}{llll}
u_{k} & u_{k} & \cdots & u_{k+H_{p}-1}
\end{array}\right]^{T}
\end{gathered}
$$

and $R$ is a symmetric positive semi-definite matrix of size $H_{p}$. Equation 1 is used to express the predicted output vector $\tilde{Y}$ in terms of the system matrices. ${ }^{1}$

$$
\begin{gathered}
K_{C A}=\left[\begin{array}{c}
C A \\
C A^{2} \\
\vdots \\
C A^{H P}
\end{array}\right] \\
K_{C A B}=\left[\begin{array}{ccccc}
C B & 0 & 0 & 0 & 0 \\
C A B & C B & 0 & 0 & 0 \\
C A^{2} B & C A B & C B & 0 & 0 \\
\vdots & \vdots & & \ddots & 0 \\
C A^{H_{p}-1} B & \cdots & C A^{2} B & C A B & C B
\end{array}\right]
\end{gathered}
$$




$$
K_{C A D}=\left[\begin{array}{c}
C D \\
C A D+C D \\
C A^{2} D+C A D+C D \\
\vdots \\
C A^{H_{p}-1} D+C A^{H_{p}-2}+\cdots+C D
\end{array}\right]
$$

Equations 6 through 8 can be substituted into Equation 2 resulting in the cost function in Equation 9 that is in terms of the system state $x_{k}$, desired trajectory $W$, control vector $U$, and system matrices $A, B, C, D$ and $R$.

$$
J=\left(W-K_{C A} X_{k}-K_{C A B} U-K_{C A D 1}\right)^{T}\left(W-K_{C A} X_{k}-K_{C A B} U-K_{C A D}\right)+U^{T} R U
$$

The control $U$, which minimizes Equation 9 is

$$
U=\mathrm{K}\left(W-K_{C A} X_{k}-K_{C A D 1}\right)
$$

where,

$$
\mathrm{K}=\left(K_{C A B}^{T}{ }^{T} K_{C A B}+R\right)^{-1} K_{C A B}^{T}
$$

Equation 10 contains the optimal control inputs over the entire control horizon, however at time $k$ only the first element $u_{k}$ is needed. The first element $u_{k}$ is extracted from Equation 10 by defining $\mathrm{K}_{1}$ as the first row of $\mathrm{K}$. The optimal control over the next time sample becomes

$$
u_{k}=\mathrm{K}_{1}\left(W-K_{C A} X_{k}-K_{C A D}\right)
$$

where, calculation of the first element of the optimal control sequence requires the desired trajectory $W$ over the prediction horizon and the current state $x_{k}$.

\section{Parafoil And Payload Model}

The combined system of the parafoil canopy and the payload is represented with 6 degrees-of-freedom (DOF) including three inertial position components of the system mass center as well as the three Euler orientation angles of the parafoil and payload system. Kinematic equations of motion for the parafoil and payload system are provided in Equations 13 and 14. 


$$
\begin{gathered}
\left\{\begin{array}{c}
\dot{x} \\
\dot{y} \\
\dot{z}
\end{array}\right\}=T^{T}\left\{\begin{array}{c}
u \\
v \\
w
\end{array}\right\} \\
\left\{\begin{array}{c}
\dot{\phi} \\
\dot{\theta} \\
\dot{\psi}
\end{array}\right\}=\left[\begin{array}{ccc}
1 & s_{\phi} t_{\theta} & c_{\phi} t_{\theta} \\
0 & c_{\phi} & -s_{\phi} \\
0 & s_{\phi} / c_{\theta} & c_{\phi} / c_{\theta}
\end{array}\right]\left\{\begin{array}{l}
p \\
q \\
r
\end{array}\right\}
\end{gathered}
$$

The matrix $T$ represents the transformation matrix from an inertial reference frame to the body reference frame.

$$
T=\left[\begin{array}{ccc}
c_{\theta} c_{\psi} & c_{\theta} S_{\psi} & -s_{\theta} \\
S_{\phi} S_{\theta} c_{\psi}-c_{\phi} S_{\psi} & S_{\phi} S_{\theta} S_{\psi}+c_{\phi} c_{\psi} & c_{\theta} S_{\phi} \\
c_{\phi} S_{\theta} c_{\psi}+s_{\phi} S_{\psi} & c_{\phi} S_{\theta} S_{\psi}-S_{\phi} c_{\psi} & c_{\phi} c_{\theta}
\end{array}\right]
$$

The common shorthand notation for trigonometric functions is employed where $\sin (\alpha) \equiv s_{\alpha}, \cos (\alpha) \equiv c_{\alpha}$ and $\tan (\alpha) \equiv t_{\alpha}$. The dynamic equations of motion are provided in Equations 16 and 17 .

$$
\begin{gathered}
\left\{\begin{array}{c}
\dot{u} \\
\dot{v} \\
\dot{w}
\end{array}\right\}=\frac{1}{m_{T}}\left(\vec{F}_{A}+\vec{F}_{W}\right)-T S_{\omega} T^{T}\left\{\begin{array}{l}
u \\
v \\
w
\end{array}\right\} \\
\left\{\begin{array}{c}
\dot{p} \\
\dot{q} \\
\dot{r}
\end{array}\right\}=I_{T}^{-1}\left(\vec{M}_{A}-S_{\omega} I_{T}\left\{\begin{array}{l}
p \\
q \\
r
\end{array}\right\}\right)
\end{gathered}
$$

where,

$$
\begin{gathered}
S_{\omega}=\left[\begin{array}{ccc}
0 & -r & q \\
r & 0 & -p \\
-q & p & 0
\end{array}\right] \\
I_{T}=\left[\begin{array}{ccc}
I_{X X} & 0 & I_{X Z} \\
0 & I_{Y Y} & 0 \\
I_{X Z} & 0 & I_{Z Z}
\end{array}\right]
\end{gathered}
$$




$$
I_{T}{ }^{-1}=\left[\begin{array}{ccc}
I_{X X I} & 0 & I_{X Z I} \\
0 & I_{Y Y I} & 0 \\
I_{X Z I} & 0 & I_{Z Z I}
\end{array}\right]
$$

The weight force vector in the body reference frame is given in Equation 21.

$$
\vec{F}_{W}=m_{T} g\left\{\begin{array}{l}
-s_{\theta} \\
s_{\phi} c_{\theta} \\
c_{\phi} c_{\theta}
\end{array}\right\}
$$

The aerodynamic forces acting at the system mass center and the aerodynamic moments about the system mass center are given in Equations 22 and 23 respectively.

$$
\begin{gathered}
\vec{F}_{A}=\frac{1}{2} \rho S V_{A}\left(C_{L 0}+C_{L \alpha} \alpha+C_{L \delta a} \delta_{a}\right)\left\{\begin{array}{c}
w \\
0 \\
-u
\end{array}\right\} \\
-\frac{1}{2} \rho S V_{A}\left(C_{D 0}+C_{D \alpha 2} \alpha^{2}+C_{D \delta a} \delta_{a}\right)\left\{\begin{array}{c}
u \\
v \\
w
\end{array}\right\} \\
\vec{M}_{A}=\frac{1}{2} \rho S \bar{c} V_{A}^{2}\left\{\begin{array}{c}
C_{l \phi} \phi+\frac{C_{l p} \bar{c} p}{2 V_{a i r}}+\frac{C_{l \delta a} \delta_{a}}{\bar{d}} \\
C_{m 0}+C_{m \alpha} \alpha+\frac{C_{m q} \bar{c} q}{2 V_{a i r}} \\
\frac{C_{n r} \bar{c} r}{2 V_{a i r}}+\frac{C_{n \delta a} \delta_{a}}{\bar{d}}
\end{array}\right\}
\end{gathered}
$$

Model predictive control requires a linear model of the states to be controlled. The desired states to control in a parafoil and payload system are the inertial positions $x$ and $y$. Equations 13 through 23 describing the parafoil and payload system are nonlinear and in order to apply standard model predictive control, must be linearized. Consider a parafoil and payload in a steady turn performing a helix as it falls. All the states excluding the inertial positions $x, y$ and $z$ and Euler yaw angle reach a steady state. The inertial positions do not appear in any of the equations of motion. However; the yaw angle appears in Equation 13 relating the inertial velocities to the body velocities. A linear model that accurately represents the inertial positions $x, y$ and $z$ of the nonlinear model must constrain the yaw angle to only small changes about a nominal yaw angle. Constraining the yaw angle in such a way 
limits the model to nearly straight flight and is not sufficient for general flight. Observation of a parafoil and payload system shows that the velocities $u, v$ and $w$ expressed in the body reference frame are nearly constant under typical flight conditions. If a reduced state $\left[\begin{array}{llll}\phi & \psi & p & r\end{array}\right]^{T}$ is considered for model predictive control purposes then the equations for $\left[\begin{array}{llll}\dot{\phi} & \dot{\psi} & \dot{p} & \dot{r}\end{array}\right]^{T}$ describing the rolling and pitching in Equations 14 and 17 can be linearized assuming that the aerodynamic velocity $V_{A}$ is constant. Euler pitch is not included in the reduced state because after linearization pitch becomes uncoupled from both rolling and yawing motion. The equations for the reduced states are linearized about the steady state in Equation 24 and given in Equation 25 with convention that $S^{*}=S-S_{0}$.

$$
S_{0}=\left\{\begin{array}{c}
\phi_{0} \\
\theta_{0} \\
p_{0} \\
q_{0} \\
r_{0} \\
\delta_{a 0}
\end{array}\right\}=\left\{\begin{array}{c}
0 \\
\theta_{0} \\
0 \\
0 \\
0 \\
0
\end{array}\right\}
$$

$$
\left\{\begin{array}{l}
\dot{\phi}^{*} \\
\dot{\psi}^{*} \\
\dot{p}^{*} \\
\dot{r}^{*}
\end{array}\right\}=\left[\begin{array}{cccc}
0 & 0 & A_{13} & 0 \\
0 & 0 & 0 & A_{24} \\
A_{31} & 0 & A_{33} & A_{34} \\
A_{41} & 0 & A_{43} & A_{44}
\end{array}\right]\left\{\begin{array}{c}
\phi^{*} \\
\psi^{*} \\
p^{*} \\
r^{*}
\end{array}\right\}+\left[\begin{array}{c}
0 \\
0 \\
B_{3} \\
B_{4}
\end{array}\right]\left\{\delta_{a}^{*}\right\}+\left[\begin{array}{c}
0 \\
0 \\
D_{1} \\
D_{2}
\end{array}\right]\left\{\delta_{\text {bias }}\right\}
$$

where,

$$
\begin{gathered}
A_{13}=1 \\
A_{24}=1 / c_{\theta_{0}} \\
A_{31}=\frac{1}{2} \rho S \bar{c} V_{\text {air }}{ }^{2} C_{l \phi} I_{X X I}
\end{gathered}
$$




$$
\begin{gathered}
A_{33}=\frac{1}{4} \rho S \bar{c}^{2} V_{a i r} I_{X X I} C_{l p} \\
A_{34}=\frac{1}{4} \rho S \bar{c}^{2} V_{a i r} I_{X Z I} C_{n r} \\
A_{41}=\frac{1}{2} \rho S \bar{c} V_{a i r}{ }^{2} I_{X Z I} C_{l \phi} \\
A_{43}=\frac{1}{4} \rho S \bar{c}^{2} V_{a i r} I_{X Z I} C_{l p} \\
A_{44}=\frac{1}{4} \rho S \bar{c}^{2} V_{a i r} I_{Z Z I} C_{n r} \\
B_{3}=\frac{\rho S \bar{c} V_{a i r}{ }^{2}\left(C_{l \delta a} I_{X X I}+C_{n \delta a} I_{X Z I}\right)}{2 \bar{d}} \\
B_{4}=\frac{\rho S \bar{c} V_{a i r}{ }^{2}\left(C_{l \delta a} I_{X Z I}+C_{n \delta a} I_{Z Z I}\right)}{2 \bar{d}} \\
D_{2}=\frac{\rho S \bar{c} V_{a i r}{ }^{2} I_{Z Z I}}{\bar{d}} \\
D_{1}=\frac{\rho S \bar{c} V_{a i r}{ }^{2} I_{X Z I}}{\bar{d}}
\end{gathered}
$$

\section{Mapping Desired X-Y Path Into Desired Yaw Angle}

The typical desired trajectories of a parafoil and payload system are points in the $x-y$ plane and according to Equation 1 the desired output must be a linear combination of the linear model states. In order to use the linear model described in Equation 25 for model predictive control the desired trajectory in the x-y plane must be mapped into a desired trajectory in terms of the reduced states $\left[\begin{array}{llll}l & \psi & p & r\end{array}\right]^{T}$. A straightforward mapping is to assume that the side velocity $v$ is small and the parafoil is traveling in the direction of its heading $\psi$. Equation 38 can then convert the desired path in the x-y plane to a desired heading. 


$$
\psi_{D}(t)=\tan ^{-1}\left(\left.\frac{d x}{d y}\right|_{t}\right)
$$

The evaluation of Equation 38 requires a continuous expression for $d x / d y$ during the prediction horizon. A technique for determining a polynomial to connect the points $\left(\left(x_{0}, y_{0}\right),\left(x_{1}, y_{1}\right) \cdots\left(x_{n}, y_{n}\right)\right)$ that describe the desired path is to use a parameter $t$ on an interval $\left[t_{0}, t_{n}\right]$, with $t_{0}<t_{1}<\cdots<t_{n}$ and construct approximations $x_{i}=x\left(t_{i}\right)$ and $y_{i}=y\left(t_{i}\right)$ for each $i=0,1, \cdots n$. The value of $t_{0}$ is the time when the prediction starts. Assuming that $V_{A}$ is constant the following times $t_{i}$ are approximated by dividing $V_{A}$ by the distance between points $\left(x_{i-1}, y_{i-1}\right)$ and $\left(x_{i}, y_{i}\right)$. The continuous path in the $x$-y plane is approximated by constructing $x_{i}=x\left(t_{i}\right)$ and $y_{i}=y\left(t_{i}\right)$ from third order Lagrange interpolating polynomials in Equations 39 through $41 .^{5}$

$$
\begin{gathered}
x\left(t_{i}\right)=\sum_{n=0}^{3} x_{n} L_{n}(t) \\
y\left(t_{i}\right)=\sum_{n=0}^{3} y_{n} L_{n}(t) \\
L_{n}=\frac{\left(t-t_{0}\right)\left(t-t_{1}\right)\left(t-t_{2}\right)\left(t-t_{3}\right)}{\left(t_{n}-t_{0}\right)\left(t_{n}-t_{1}\right)\left(t_{n}-t_{2}\right)\left(t_{n}-t_{3}\right)}
\end{gathered}
$$

The continuous time derivatives of $x_{i}=x\left(t_{i}\right)$ and $y_{i}=y\left(t_{i}\right)$ are given in Equations 42 through 47.

$$
\begin{gathered}
\frac{d x}{d t}=\sum_{n=0}^{3} x_{n} D_{n} \\
\frac{d y}{d t}=\sum_{n=0}^{3} y_{n} D_{n} \\
D_{0}=\frac{\left(t-t_{1}\right)\left(t-t_{2}\right)+\left(t-t_{1}\right)\left(t-t_{3}\right)+\left(t-t_{2}\right)\left(t-t_{3}\right)}{\left(t_{0}-t_{1}\right)\left(t_{0}-t_{2}\right)\left(t_{0}-t_{3}\right)} \\
D_{1}=\frac{\left(t-t_{0}\right)\left(t-t_{2}\right)+\left(t-t_{0}\right)\left(t-t_{3}\right)+\left(t-t_{2}\right)\left(t-t_{3}\right)}{\left(t_{1}-t_{0}\right)\left(t_{1}-t_{2}\right)\left(t_{1}-t_{3}\right)} \\
D_{2}=\frac{\left(t-t_{0}\right)\left(t-t_{1}\right)+\left(t-t_{0}\right)\left(t-t_{3}\right)+\left(t-t_{1}\right)\left(t-t_{3}\right)}{\left(t_{2}-t_{0}\right)\left(t_{2}-t_{1}\right)\left(t_{2}-t_{3}\right)}
\end{gathered}
$$




$$
D_{3}=\frac{\left(t-t_{0}\right)\left(t-t_{1}\right)+\left(t-t_{0}\right)\left(t-t_{2}\right)+\left(t-t_{1}\right)\left(t-t_{2}\right)}{\left(t_{3}-t_{0}\right)\left(t_{3}-t_{1}\right)\left(t_{3}-t_{2}\right)}
$$

Equation 38 can be solved for all time using Equation 48.

$$
\left.\frac{d x}{d y}\right|_{t}=\left.\frac{d x / d t}{d y / d t}\right|_{t}
$$

\section{Test System}

The parafoil and payload system used in all testing is shown in Figures 1 through 3 with the physical parameters in Table 1. A test flight commences by launching the system from the ground, a 10-inch propeller powers the test system to altitudes of 250 to $400 \mathrm{ft}$ where the propeller is stopped and gliding commences, lasting approximately 20 seconds for every 100 feet of altitude.

Full state measurement of the parafoil required in the optimal control sequence is achieved through a sensor package that includes three single axis gyroscopes a three-axis accelerometer and a three-axis magnetometer shown in Figure 3. Inertial positions $x$ and $y$ required in the mapping of the desired $x-y$ path into a desired yaw angle are obtained from a Wide Area Augmentation System (WAAS) enabled Global Positioning Satellite (GPS) receiver shown in Figure 1. The sensors are supplemented with a wireless transceiver that transmits data from the parafoil and receives commands during flight. An operator controlled transmitter switches control of the parafoil to one of three modes: manual, estimation or autonomous. Manual mode allows the operator to manually fly the parafoil. Estimation mode allows estimation of linear model aerodynamic coefficients required for model predictive control. Autonomous mode controls the parafoil using the optimal control calculated from the model predictive control law.

\section{Identification Of Aerodynamic Coefficients}

Application of the reduced order model requires the knowledge of five constant aerodynamic coefficients:

$C_{l \phi}, C_{l p}, C_{l \delta a}, C_{n r}, C_{n \delta a}$ and the constant bias term $\delta_{b i a s}$. The six parameters are estimated using recursive weighted least-squares estimation where, $\vec{Z}_{i}$ are measurements, $\vec{x}_{i}$ are parameters to be estimated and $\vec{n}_{i}$ is zero mean measurement noise. 


$$
\vec{z}_{i}=H_{i} \vec{x}+\vec{n}_{i}
$$

The recursive weighted least squares estimation to Equation 49 is given in Equations 50 and 51 where, $P_{i}$ is the error covariance estimate of the parameters at measurement $i$ and $Q$ is the measurement noise covariance. ${ }^{6}$

$$
\begin{gathered}
\hat{x}_{i}=\hat{x}_{i-1}+P_{k} H_{k}{ }^{T} Q^{-1}\left(z_{k}-H_{k} \hat{x}_{k}\right) \\
P_{i}=P_{i-1}-P_{i-1} H_{i}{ }^{T}\left(Q+H_{i} P_{i-1} H_{i}{ }^{T}\right)^{-1} H_{i} P_{i-1}
\end{gathered}
$$

The matrix $H_{i}$ giving a linear relationship between the parameters to be estimated and measurements is acquired by linearizing $\dot{p}^{*}$ and $\dot{r}^{*}$ in equation 26 and is given in Equation 52 .

$$
H_{i}=\frac{\rho S \bar{C} V_{\text {air }}{ }^{2}}{2}\left[\begin{array}{cc}
I_{X X I} \phi_{i} & I_{X Z I} \phi_{i} \\
I_{X X I} \frac{\bar{c} p_{i}}{2 V_{\text {air }}} & I_{X Z I} \frac{\bar{c} p_{i}}{2 V_{\text {air }}} \\
I_{X X I} \frac{\delta_{a}}{\bar{d}} & I_{X Z I} \frac{\delta_{a}}{\bar{d}} \\
I_{X Z I} \frac{\bar{c} r_{i}}{2 V_{\text {air }}} & I_{Z Z I} \frac{\bar{c} r_{i}}{2 V_{\text {air }}} \\
I_{X Z I} \frac{\delta_{a}}{\bar{d}} & I_{Z Z I} \frac{\delta_{a}}{\bar{d}} \\
\frac{I_{X Z I}}{\bar{d}} & \frac{I_{Z Z I}}{\bar{d}}
\end{array}\right]^{T}
$$

The recursive weighted least squares estimation requires the differentiation of the measured roll and yaw rates. The control sequence shown in Figure 4 and used in parameter identification was chosen to be sinusoidal in order to ensure that numerical differentiation of roll and yaw rates produced significant signals. Figure 5 shows differentiation of measured roll and yaw rates. The recursive weighted least squares estimation is initialized with $P_{1}$ as a 6 by 6 diagonal matrix with 0.05 along the diagonal, $C_{l \phi}, C_{l p}, C_{l \delta a}, C_{n r}, C_{n \delta a}$ and $\delta_{b i a s}$ as -0.1, -0.5, 0.1, $0.1,0.1$ and 0.0 respectively. The measurement noise covariance $Q$ was set as a 2 × 2 diagonal matrix with $Q_{1,1}=0.00475$ and $Q_{2,2}=0.0005$. The aerodynamic parameter estimations are shown in Figures 6 and 7 with the final estimates of $C_{l \phi}, C_{l p}, C_{l \delta a}, C_{n r}, C_{n \delta a}$ and $\delta_{\text {bias }}$ given in Table 2. being -0.0244, -0.0320, 0.0050, $0.0501,0.0014$ and -0.00017 . 
The discrete time linear reduced order model is simulated with 1 second sampling, estimated aerodynamic coefficients and the control sequence in Figure 4. The model results are compared to the measured states of the test system in Figures 8 through 11. The reduced order model is able to capture the fundamental dynamics of the parafoil and payload. A bias in the body yaw rate of $2.5 \mathrm{deg} / \mathrm{sec}$ is visible in Figure 8.

\section{Model Predictive Control Results}

The prediction of desired heading angle with third order Lagrange interpolating polynomials in Equation 38 requires four desired path points. The first point is defined as the current position of the parafoil and payload system during implementation of the controller. The second point is defined as the location along the desired path that is a distance $\sigma$ ahead of the current position and called the intersect parameter. The third and fourth points are the next two desired path points. Figure 12 shows a desired path and the Lagrange interpolating polynomial found from Equations 38 through 48. The update rate of the model predictive controller was chosen to be 1 second and the linear model is converted to a discrete time system of the form in Equation 1 with a sampling period of $1 \mathrm{sec}$. The discrete time system matrices $A, B, C$ and $D$ required for the model predictive controller are given in Equations 53-56.

$$
\begin{aligned}
& A=\left[\begin{array}{cccc}
0.4251 & 0 & 0.7677 & 0.0047 \\
0.0551 & 1.0000 & 0.0236 & 0.7059 \\
-1.0084 & 0 & 0.3713 & 0.0070 \\
0.0823 & 0 & 0.0593 & 0.4733
\end{array}\right] \\
& B=\left[\begin{array}{l}
0.0573 \\
0.0945 \\
0.1010 \\
0.1693
\end{array}\right] \\
& C=\left[\begin{array}{llll}
0 & 1 & 0 & 0
\end{array}\right] \\
& D=\left[\begin{array}{c}
0.0002 \\
-0.0122 \\
0.0003 \\
-0.0216
\end{array}\right]
\end{aligned}
$$


The matrix $R$ penalizing control magnitude in the optimal control sequence is selected as an $H_{p} \times H_{p}$ matrix with 0.35 on the diagonal and zeros everywhere else. The test parafoil system with the model predictive control law is simulated with prediction horizons of 2,3,4,5,10 and 20 and shown in Figures 13 and 14. Tracking error is decreased as the prediction horizon $H_{p}$ is increased from 2 to 10 , however, as $H_{p}$ is further increased from 10 to 20 no change in performance is observed and $H_{p}$ is selected to be 10 for the test system control law. Results for the model predictive controller are shown in Figures 15 through 20 with $H_{p}=10$ and $\sigma=100 \mathrm{ft}$. Figures 15 and 16 show the measured path of the parafoil and payload compared to a desired straight path and control with no wind. Control is initiated at the origin with the parafoil and payload initially traveling away from the desired path and $40 \mathrm{ft}$ off line. The initial control response is large and negative corresponding to left braking and negative cross range. The parafoil has a maximum error of 75 feet at $100 \mathrm{ft}$ down range then overshoots the desired by path by 39 feet at 510 feet down range before a final error of 9 feet at impact.

Figures 17 and 18 show the measured path of the parafoil and payload compared to the desired straight path and control with a $12 \mathrm{ft} / \mathrm{sec}$ cross wind from positive to negative cross range. Control is again initiated at the origin with the parafoil and payload initially traveling away from the desired path and $100 \mathrm{ft}$ off line. The parafoil has a similar oscillatory response with a maximum error of 119 feet at $230 \mathrm{ft}$ down range as it overshoots the desired path. The parafoil turns back towards the desired path and comes within 18 feet before the wind pushes it further away. The final error at impact is 6 feet. The larger error from the crosswind is due to the difference in measured yaw angle and heading angle because of parafoil sideslip. Figures 19 and 20 show the performance of the model predictive controller when tracking the more complicated S-shaped path. Control is initiated when the parafoil and payload are $210 \mathrm{ft}$ offline. The maximum error during the flight is $45 \mathrm{feet}$ at 550 feet down and $-550 \mathrm{ft}$ cross range. The model predictive controller is able to predict the required control input so that the parafoil and payload system are able to achieve close proximity to the desired points as they are passed.

\section{Conclusions}

A model predictive control strategy was developed for a parafoil and payload system. To support the flight control law, a reduced state linear model was created that uses roll angle, yaw angle, body roll rate, and body yaw 
rate of the parafoil and payload system. Application of the reduced order model requires the knowledge of five constant aerodynamic coefficients: $C_{l \phi}, C_{l p}, C_{l \delta a}, C_{n r}, C_{n \delta a}$ and a constant bias term $\delta_{\text {bias }}$. A recursive weighted least squares estimation is used to estimate the six parameters. The estimated parameters and reduced state model is compared with flight data and it was shown that they adequately modeled the parafoil and payload system. In order to use the reduced state linear model, the desired $x-y$ trajectory is mapped into desired yaw angles using Lagrange interpolating polynomials assuming a constant aerodynamic velocity. Three exemplar flight tests are used to show that model predictive control is an effective way to autonomously control the trajectory of a parafoil and payload system. Model predictive control is a natural way to control a parafoil and payload because it mimics the process that a pilot controlling a paraglider estimates both the path and control sequence to achieve a desired outcome.

\section{References}

${ }^{1}$ Ikonen, E., Najim, K., Advanced Process Identification and Control, Marcel Dekker Inc., New York, 2002.

${ }^{2}$ Mei, G., Kareem, A., "Model Predictive Control of Wind-Excited Building: Benchmark Study.” Journal Of Engineering Mechanics., vol. 130, pp. 459-465, Apr. 2004.

${ }^{3}$ Tsai, C. C., Huang, C. H., "Model Reference Adaptive Predictive Control for a Variable-Frequency OilCooling Machine.” IEEE Transactions On Industrial Electronics., vol. 51, No. 2, pp. 330-339, Apr. 2004.

${ }^{4}$ Kvaternik, Piatak, Nion, Langston, Singleton, Bennett, Brown, “An experimental evaluation of generalized predictive control for tiltrotor aeroelastic stability augmentation in airplane mode of flight." Journal of the American Helicopter Society, v 47, n 3, July, 2002, p 198-208

${ }^{5}$ Faires, Burden, Numerical Methods, PWS Publishing Company, Boston, 1993.

${ }^{6}$ DeRusso, P., Roy, R., Close C., Desrochers A., State Variables for Engineers, John Wiley and Sons, Inc., New York, 1998. 


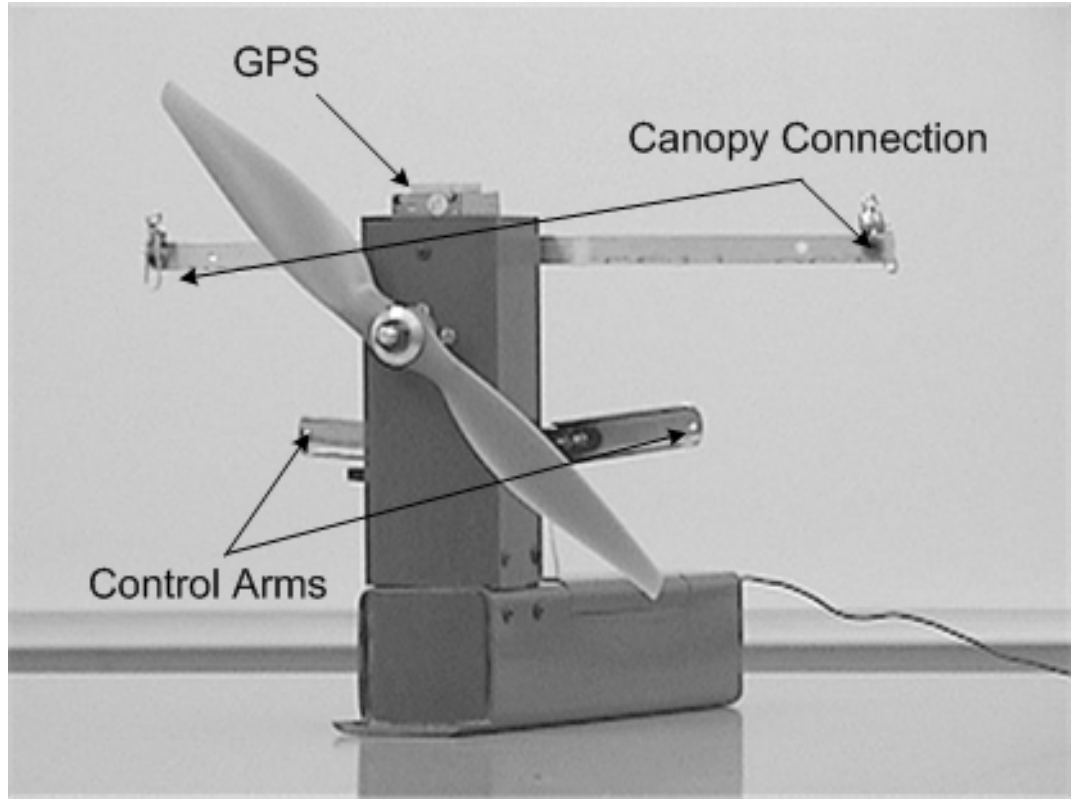

Fig. 1. Payload 


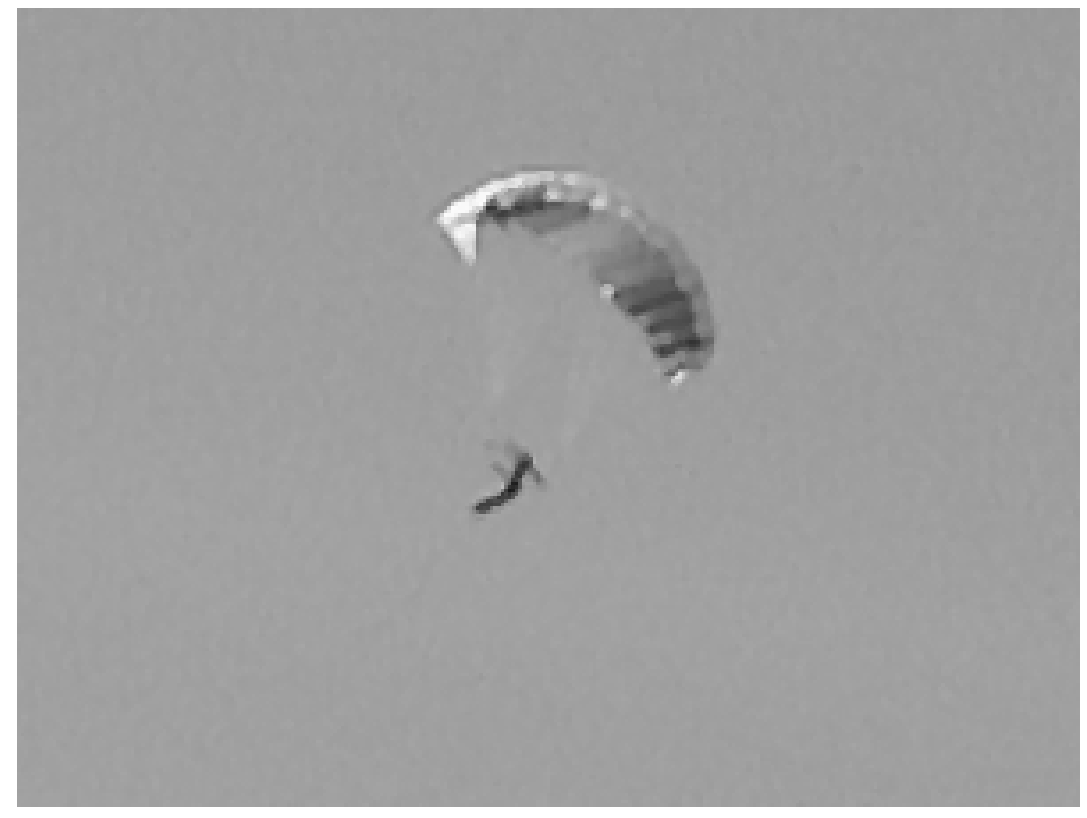

Fig. 2. Parafoil And Payload System 


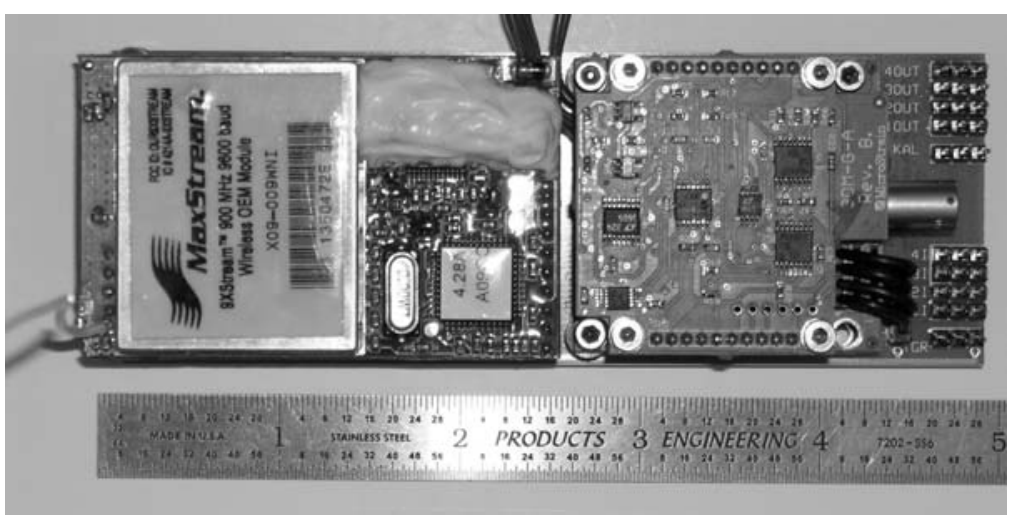

Fig. 3. Parafoil And Payload System 


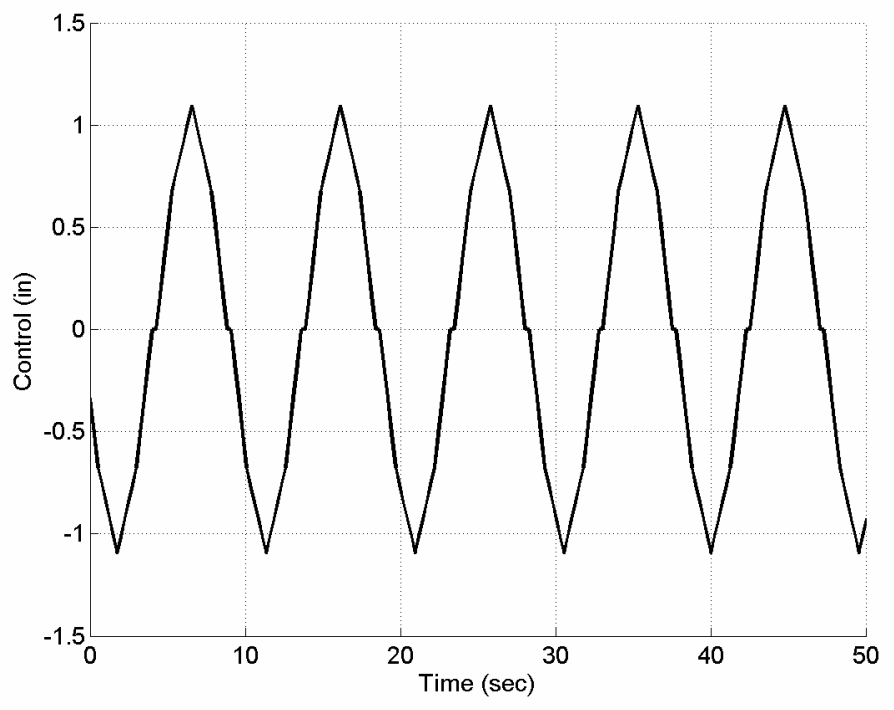

Fig. 4. Control Sequence 


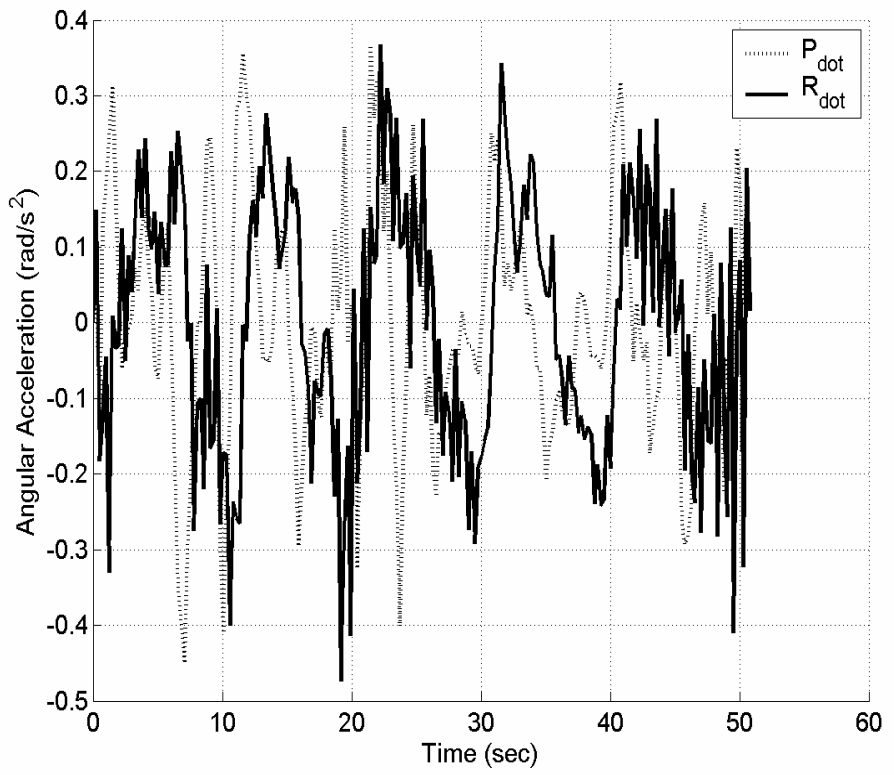

Fig. 5. Differentiated Measured Body Roll and Yaw Rates 


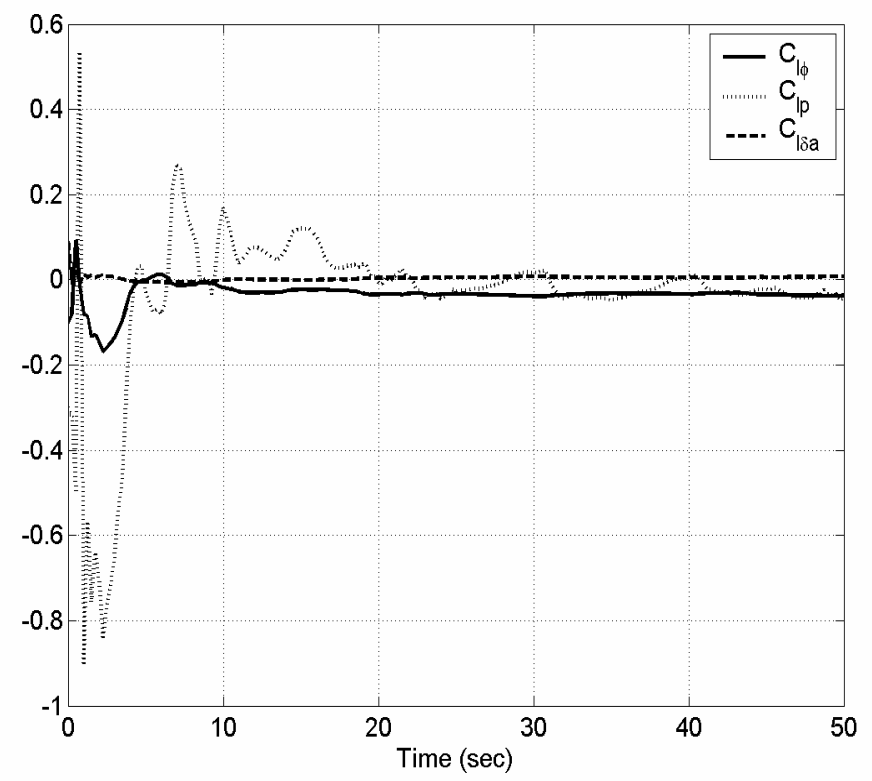

Fig. 6. Estimated Roll Aerodynamic Coefficients 


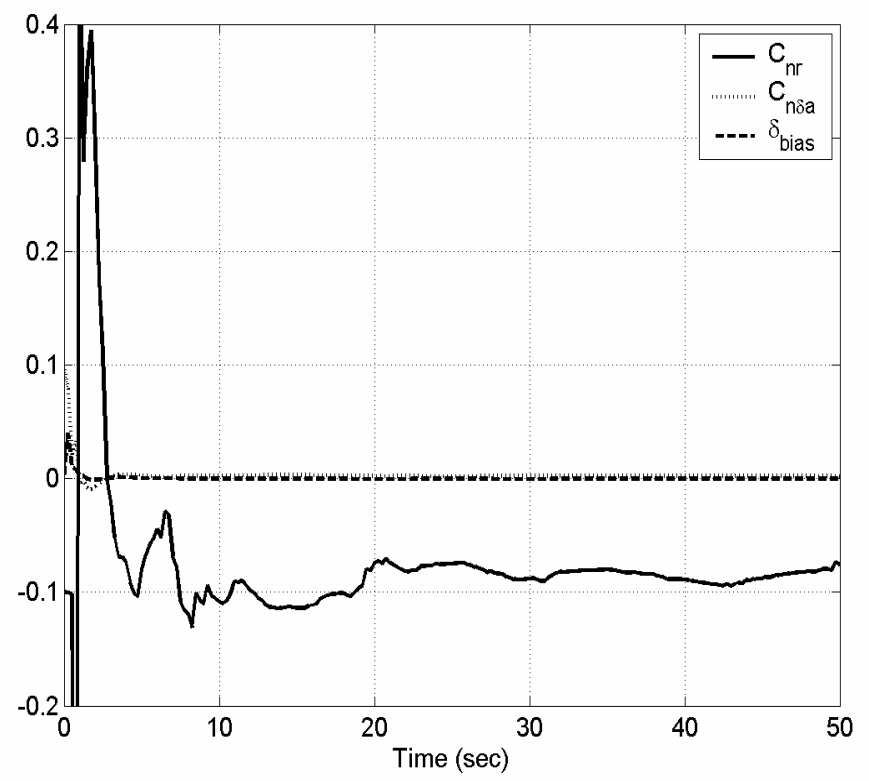

Fig. 7. Estimated Yaw Aerodynamic Coefficients and Bias 


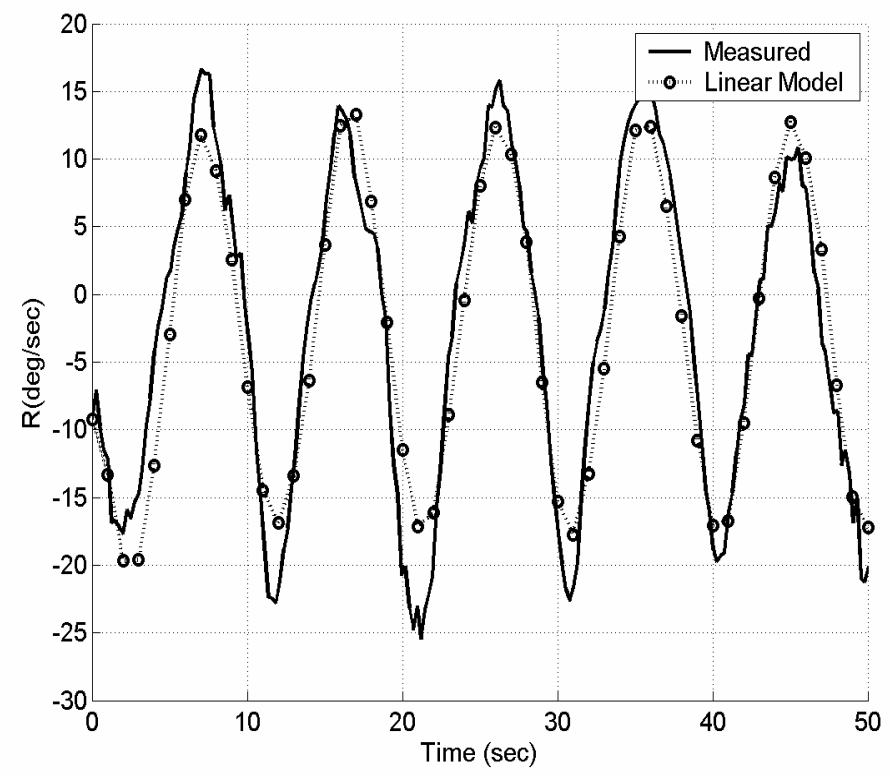

Fig 8. Comparison of Measured and Model Yaw Rate 


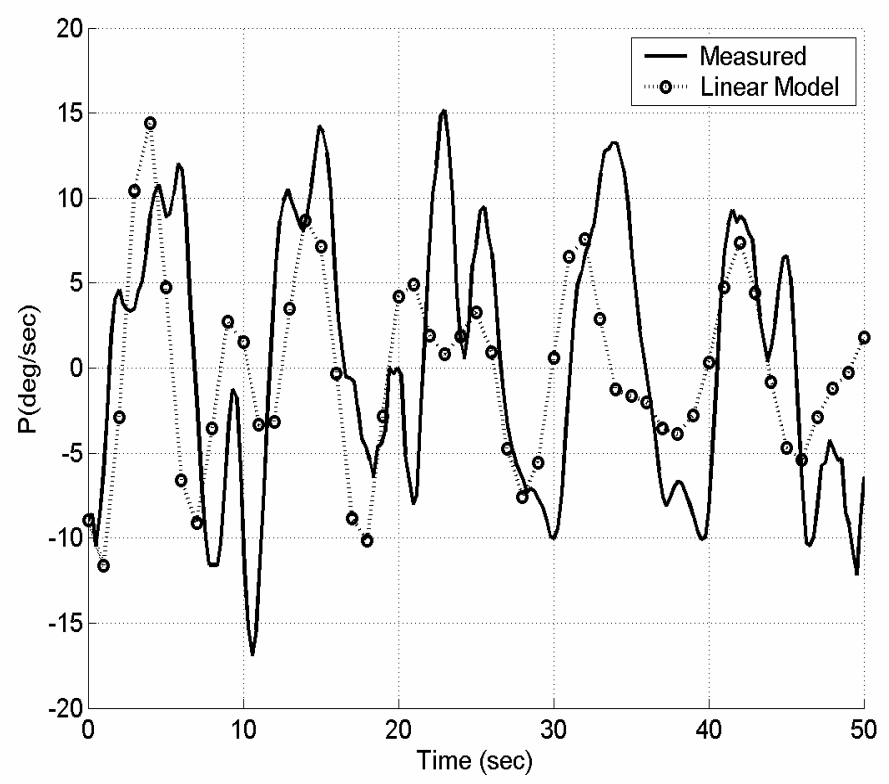

Fig. 9. Comparison of Measured and Model Roll Rate 


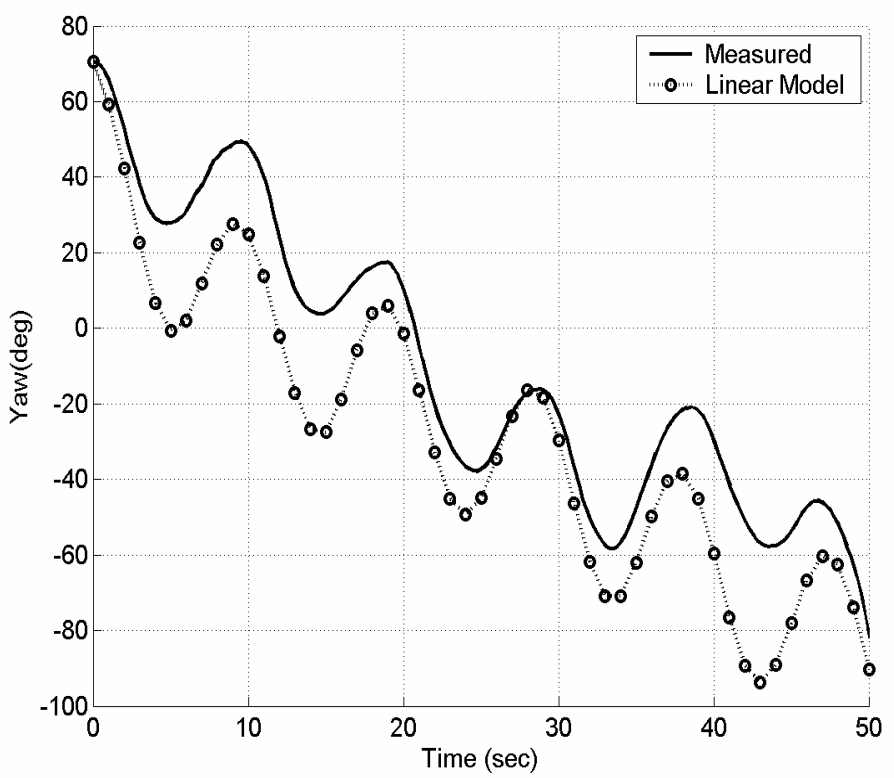

Fig. 10. Comparison of Measured and Model Yaw Angle 


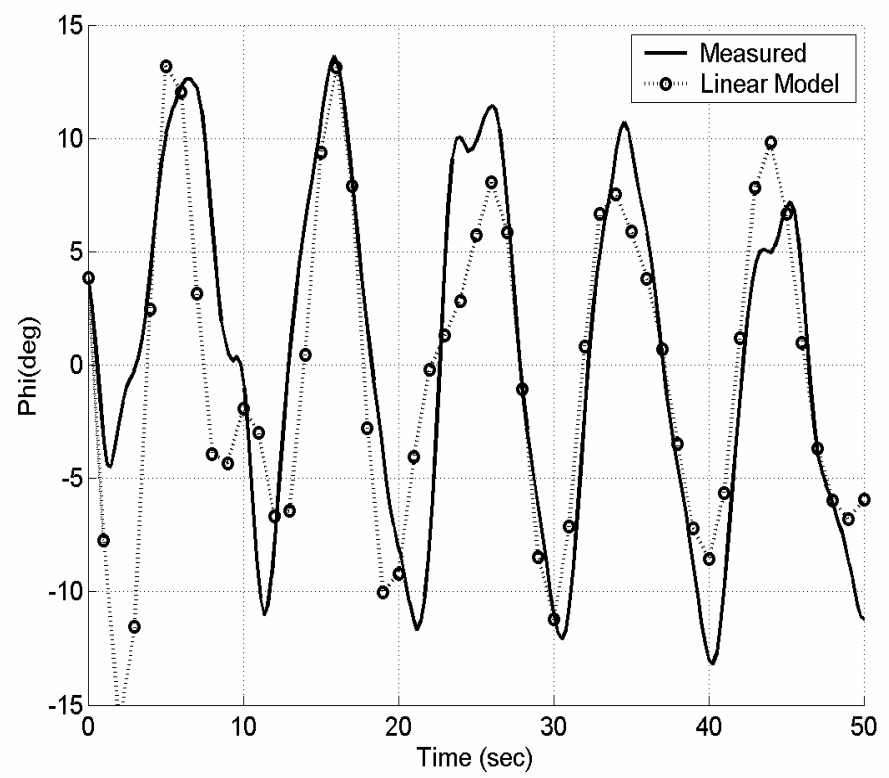

Fig. 11. Comparison of Measured and Model Roll Angle 


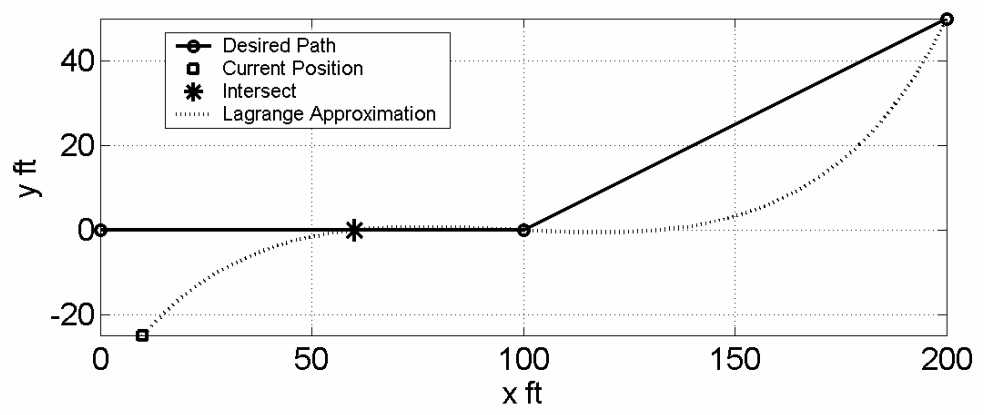

Fig. 12. Lagrange Approximating Polynomial 


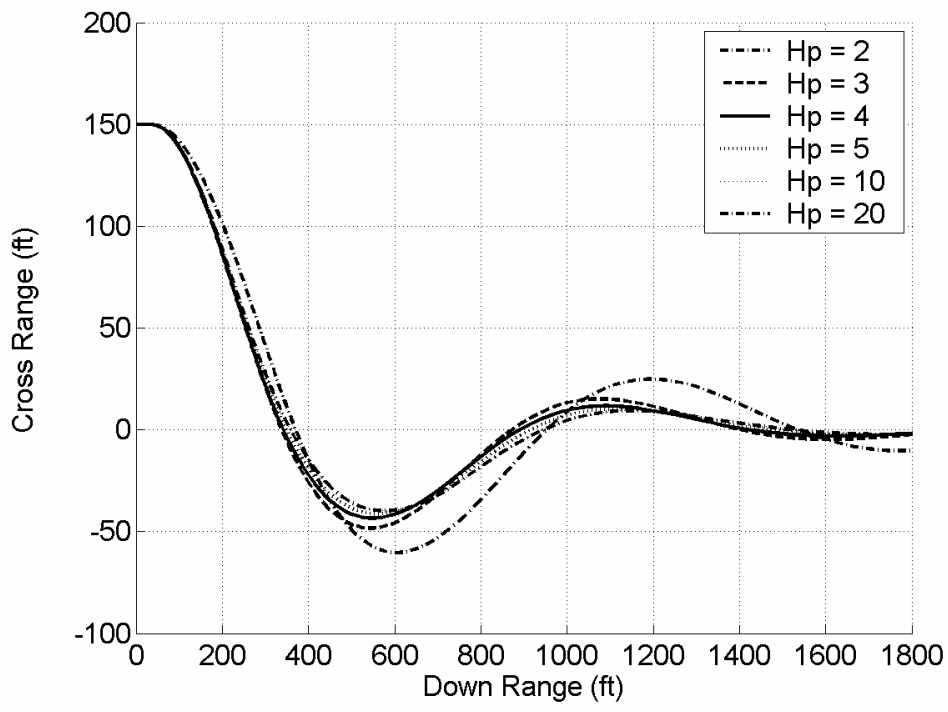

Fig 13. Simulated Tracking Of Zero Cross Range For Varying Prediction Horizons 


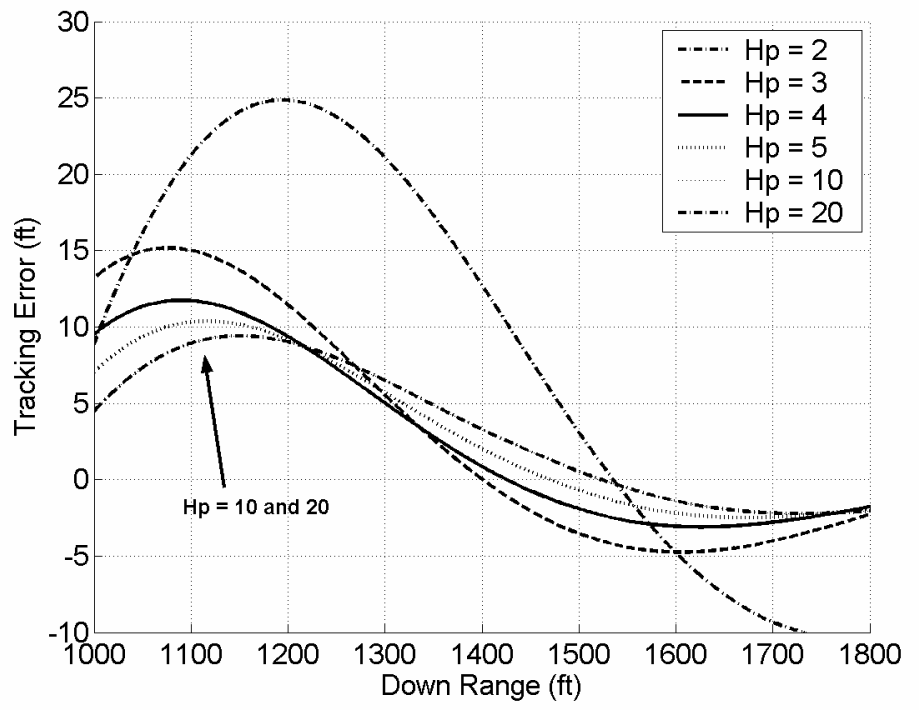

Fig. 14. Tracking Error Over Final 800 feet 


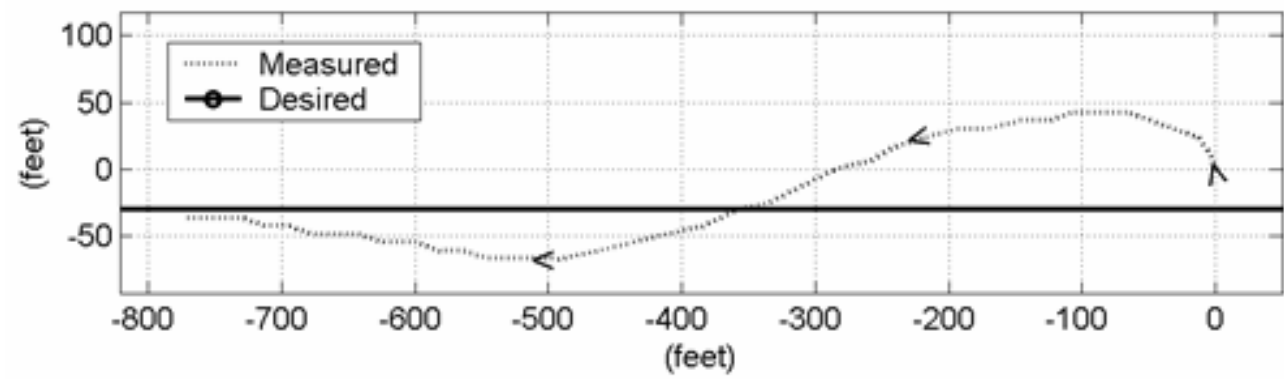

Fig. 15. Controlled Straight Path With No Wind 


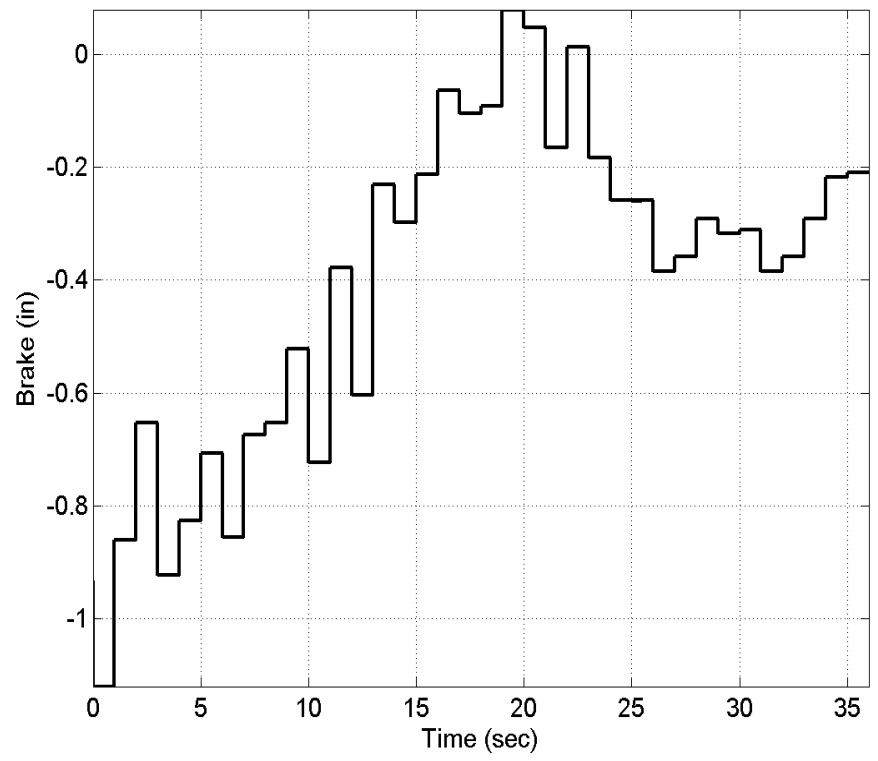

Fig. 16. Straight Path Control Input With No Wind 


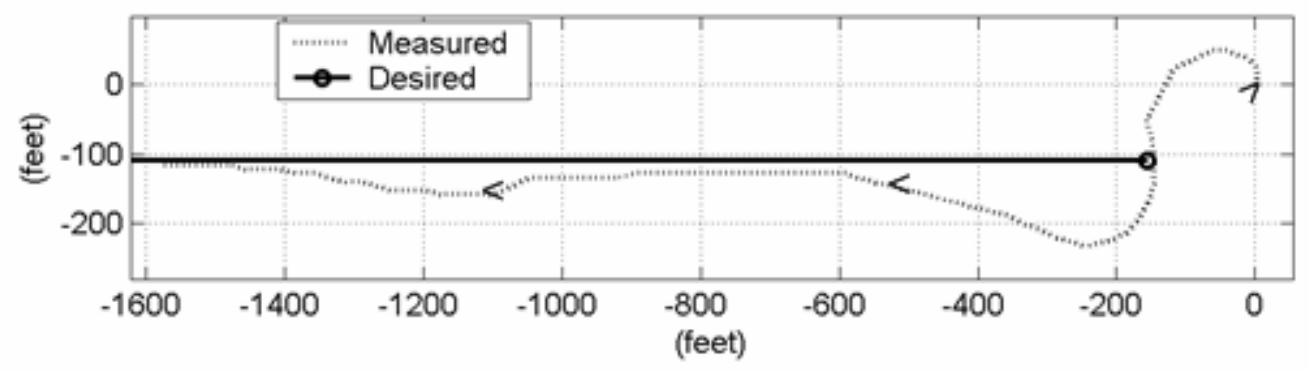

Fig. 17. Controlled Straight Path With Cross Wind 


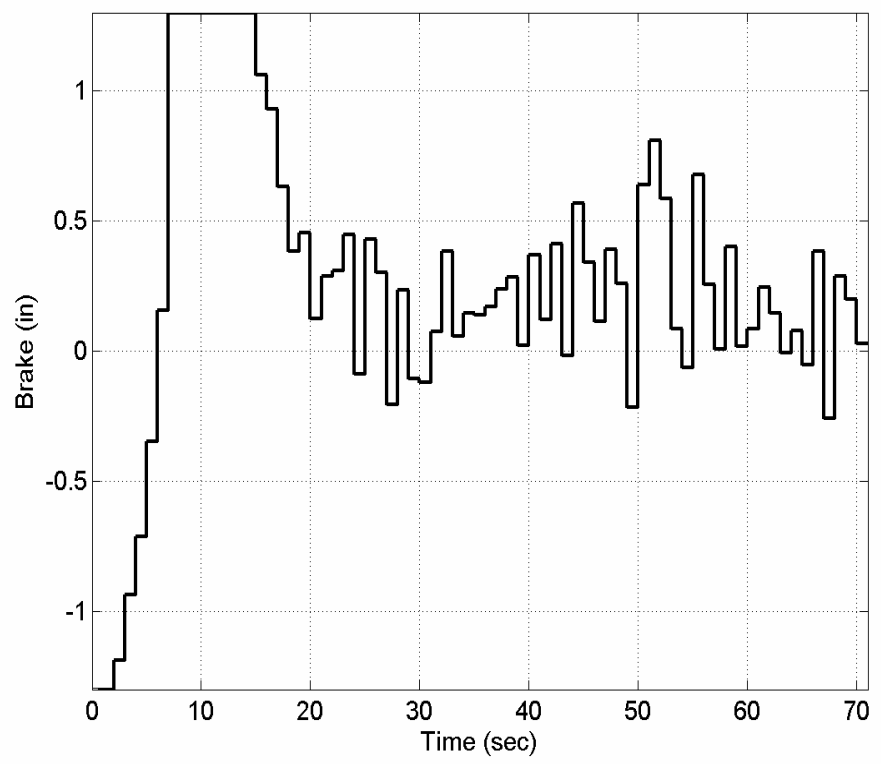

Fig 18. Straight Path Control Input With Cross Wind 


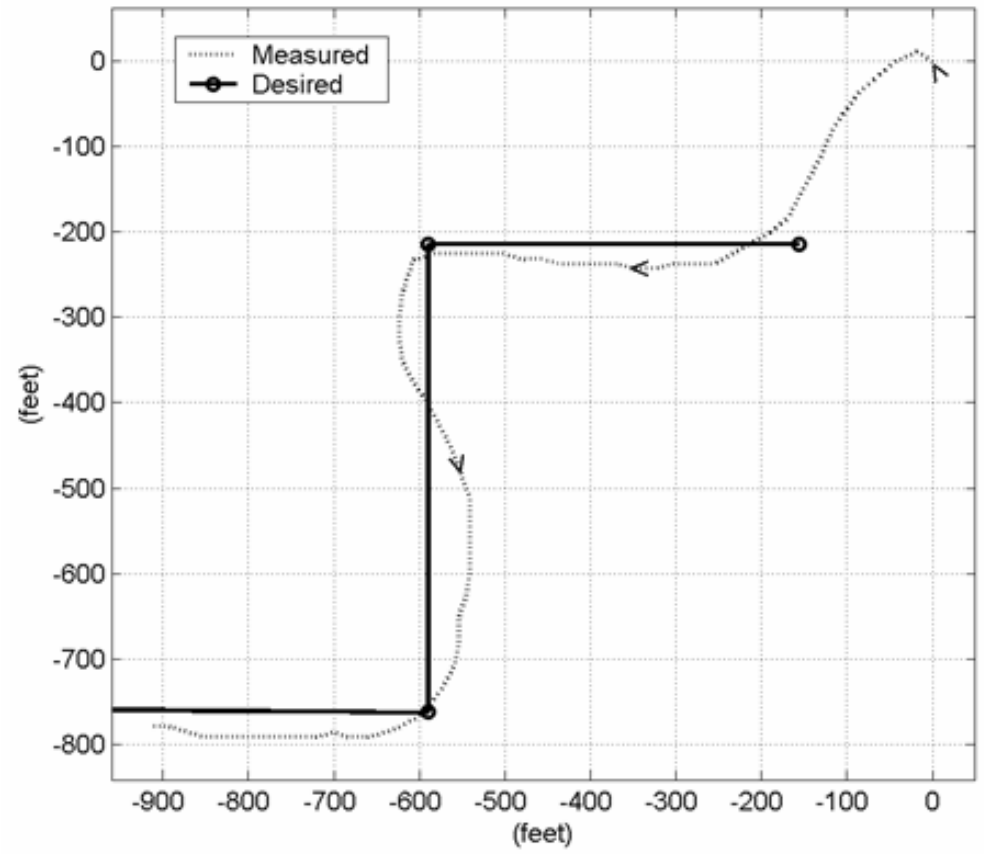

Fig. 19. Controlled "S" Path With No Wind 


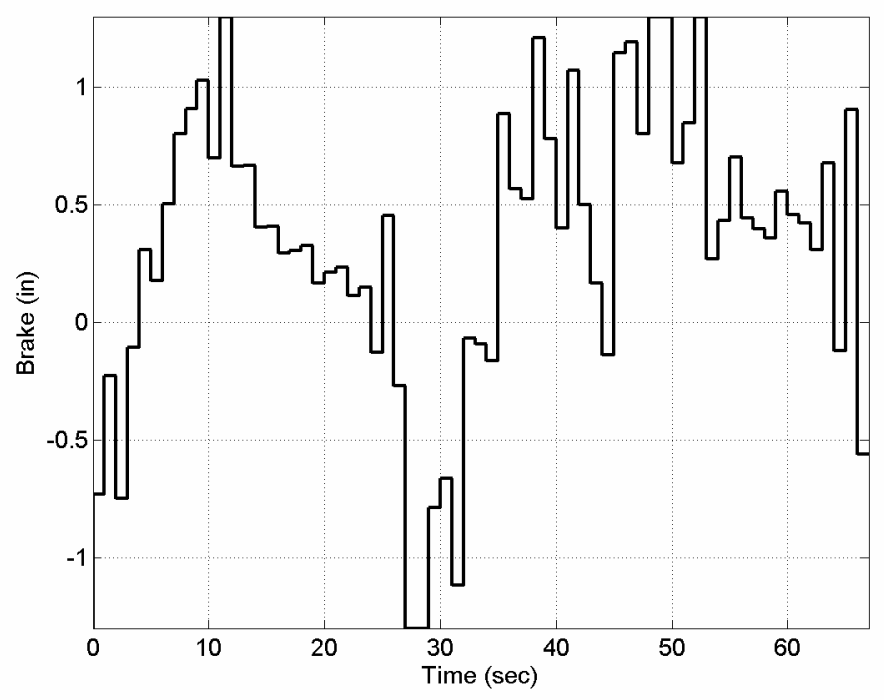

Fig. 20. "S" Path Control Input With No Wind 
Table 1. Parafoil and Payload Physical Parameters

\begin{tabular}{|l|l|l|}
\hline Variable & Value & Units \\
\hline$\rho$ & 0.0023784722 & $\mathrm{Slug} / \mathrm{ft} \wedge 3$ \\
\hline Weight & 2.0 & $\mathrm{lbf}$ \\
\hline$S$ & 7.5 & $\mathrm{ft} \wedge 2$ \\
\hline $\bar{C}$ & 1.75 & $\mathrm{ft}$ \\
\hline $\bar{d}$ & 2.0 & $\mathrm{ft}$ \\
\hline Ixx & 0.1357 & Slug-ft $\wedge 2$ \\
\hline Iyy & 0.1506 & Slug-ft $\wedge 2$ \\
\hline Izz & 0.0203 & Slug-ft $\wedge 2$ \\
\hline Ixz & 0.0025 & Slug-ft $\wedge 2$ \\
\hline Ixxi & 7.3845 & $1 /($ Slug-ft $\wedge 2)$ \\
\hline Iyyi & 6.6423 & $1 /($ Slug-ft $\wedge 2)$ \\
\hline Izzi & 49.442 & $1 /($ Slug-ft $\wedge 2)$ \\
\hline Ixzi & -0.9032 & $1 /($ Slug-ft $\wedge 2)$ \\
\hline Vair & 21.6 & $\mathrm{ft} /$ sec \\
\hline
\end{tabular}


Table 2. Estimated Model Coefficients

\begin{tabular}{|c|c|}
\hline$C_{l \phi}$ & -0.0244 \\
\hline$C_{l p}$ & -0.0320 \\
\hline$C_{l \delta a}$ & 0.0050 \\
\hline$C_{n r}$ & -0.0501 \\
\hline$C_{n \delta a}$ & 0.0014 \\
\hline$\delta_{\text {bias }}$ & -0.00017 \\
\hline
\end{tabular}

